\title{
Learning Resource Recommendation Method based on Fuzzy Logic
}

\author{
Li Guangquan $^{1,2, *}$, Xia Jiali ${ }^{1}$ and Mei Siyu ${ }^{3}$
}

\author{
${ }^{1}$ School of Information Management, Jiangxi University of Finance and Economics, Nanchang 330032, China \\ ${ }^{2}$ School of Computer and Information Engineering, Jiangxi Agriculture University, Nanchang 330045, China
}

${ }^{3}$ UCD school of computer science, University College Dublin, Belfield, Dublin 4, Ireland

Received 2 August 2018; Accepted 30 September 2018

\begin{abstract}
A learning resource recommendation method based on fuzzy logic (RRMF) was proposed to solve the ambiguity problem of categorizing learning resources and determining learners' knowledge levels in the learning resource recommendation process and to improve the accuracy of recommending learning resources. First, learning resources were modeled using fuzzy set theory. The learning resources were divided in accordance with knowledge points, and the association of each learning resource to different knowledge points was calculated. One learning resource is associated with multiple knowledge points at different degrees. Second, the learners were modeled through fuzzy cognitive diagnosis method by determining the knowledge level of learners. On this basis, a learning resource recommendation model was constructed. Scores of candidate learning resources of the target learners were predicted, and learning resources with scores that satisfy the value range were recommended to learners. Finally, the proposed RRMF was verified through a test on real datasets FrcSub, Math1, and Math2. Experimental results demonstrate that the mean absolute errors of the proposed RRMF on different datasets are $0.3045,0.2944$, and 0.2817. The proposed RRMF is remarkably superior to the recommendations based on cognitive diagnosis, probabilistic matrix factorization, and collaborative filtering. Moreover, the proposed RRMF increases the accuracy of recommendation. This finding confirms that the proposed RRMF can solve the fuzziness problem in the learning resource recommendation process effectively and increase the accuracy of the learning resource recommendation. The conclusions in this study provide novel methods for designing learning resource recommendation services.
\end{abstract}

Keywords: E-learning, Recommender systems, Cognitive diagnosis, Fuzzy sets

\section{Introduction}

Currently, learning can be completed whenever and wherever possible given the popularization of online learning. Learners can easily acquire various network learning resources in multiple ways. Numerous learning resources are published online, thereby resulting in the explosive growth of learning resources. This phenomenon increases the difficulties for learners to find the learning resources that truly satisfy their requirements. Under this circumstance, "information overload" intensifies gradually, which results in an increasingly prominent "learning loss." This problem can be effectively addressed if personalized intelligent push services are offered online, and the recommendation system must help learners find the learning resources that satisfy their own demands [1]. The key to a successful implementation of online learning is by designing an intelligent learning resource recommendation method. However, learning resource recommendations differ from conventional commodity and movie recommendations. The analysis shows that learning resource recommendation is frequently accompanied by the following ambiguities:

(1) Fuzziness of learning resource division. The division of learning resources is slightly ambiguous. Generally, the

*E-mail address: guangquanli2000@163.com

ISSN: 1791-2377 (C) 2018 Eastern Macedonia and Thrace Institute of Technology. All rights reserved. doi:10.25103/iestr.114.19 relationship between learning resources and knowledge points is not av problem of existence but of depth. One learning resource may be associated with multiple knowledge points with different degrees of association. In particular, one learning resource may be strongly associated with a knowledge point but may be weakly associated with others. Such "strength" and "weakness" are fuzzy.

(2) Fuzziness of the knowledge level of learners. The knowledge point mastery degree of learners cannot be simply defined as mastered or not. Alternatively, it shall emphasize the degree of mastery, that is, the knowledge points that learners mastered and did not master well. The degrees of mastery described as "relatively good" and "very good" are fuzzy assessments of the degree of mastery.

These fuzzy problems in learning resource recommendation may affect the accuracy of learning resource and learner models, and thus these problems may further influence the accuracy of the final recommendation. Existing methods for learning resource recommendation rarely consider such fuzzy problems. However, existing associated studies have focused on the interests and knowledge level of learners or on conventional commodity recommendation techniques [2, 3]. These recommendation methods fail to accurately construct learning resource and learner models and cannot effectively solve the fuzzy problems in learning resource recommendation. Thus, learning resource and learner models are constructed using fuzzy set theory and fuzzy cognitive diagnosis method, respectively. On this basis, the learning resource 
recommendation model is constructed to recommend appropriate learning resources to learners. The accuracy of learning resource recommendation in online learning must be improved to solve the fuzzy problems in learning resource recommendation because it may improve learning efficiency and user satisfaction.

\section{State of the art}

Currently, designing the learning resource recommendation method mainly focuses on the following three aspects:

(1) Collaborative filtering is used more extensively than other conventional commodity recommendation methods. Chen et al. [4] proposed a learning resource recommendation method, which first recognized the learning resources that learners were interested in on the basis of item-based collaborative filtering. Second, the method was used to determine the learning order of resources through sequential pattern mining algorithm and finally recommend the arranged learning resources to the learners. The experimental results verified that such a method can achieve relatively favorable recommendation performances. Rodriguez et al. [5] proposed a student-centered hybrid recommender system to accomplish the learning resource recommendation by combining collaborative filtering, content-based recommendation, and knowledge-based recommendation. dos Santos et al. [6] reported that clustering learning objects before the use of collaborative filtering techniques can improve the recommendations performance. The advantage of collaborative filtering is the free construction of learning resource and learner models. However, the recommendation performances deteriorate given the intensifying data sparsity and cold start of users in actual application scenes.

(2) Learning resources are recommended in accordance with the interest and knowledge level of learners. KlašnjaMilićević et al. [7] automatically recommended learning contents to learners by combining the interests, knowledge level and learning style of learners. Learners can improve the efficiency of learning by using this recommender system. This system divides the knowledge level of learners into five ratings, which do not reflect the knowledge point mastery degree of learners. $\mathrm{Lu}$ [8] determined the demands of learners through a multi-attribute evaluation method and recommended the desired learning resources through a fuzzy matching method. This method lacks the construction of learning resource and learner models, and its experimental effect must be further verified. Wan et al. [9] proposed a learning resource recommendation method on the basis of the mixed concept map and immune algorithm. This method considered the interest and emotional factors of learners comprehensively when recommending learning resources. However, emotion and other information are difficult to determine. Chen et al. [10] presented a recommendation method by combining two-phase blue-red trees of rule-space model and optimized learning path. This method determined the concept of learning for learners and recommended associated learning resources. This method aptly recommended a learning path. Liu et al. [11] constructed a learner model using an interest graph and proposed a learning resource recommendation method based on this interest graph. A course type was determined initially, and then relevant learning resources in accordance with the interests of learners were recommended. However, this method failed to delineate the relationship between learning resources and knowledge points. Bernacki [12] introduced a learning resource recommendation method, which considered content, difficulty, and type of learning resources comprehensively. The method recommended the optimal learning resources for learners using an analytic hierarchy process; however, it lacked the corresponding experimental analysis, and the application effect of this method should be further tested. Salehi et al. [13] developed a hybrid learning resource recommendation method, which recommended appropriate learning resources to learners on the basis of their preference tree. However, this method did not construct a learning resource model. Although these recommendation methods can recommend learning resources to learners in accordance with their states, none of these methods achieved an accurate model for learning resources and learners.

(3) Learning resources are recommended in accordance with fuzzy set theory. Wu et al. [14] suggested a learning resource recommendation method on the basis of fuzzy tree matching. In this method, the learning activity and learner models were constructed using a fuzzy tree, and a similarity calculation method based on the fuzzy tree was designed. Learning resources were recommended in accordance with the matching degree of learning resources and learners. The experimental results confirmed that this method can increase the accuracy of recommendation. However, this method overlooked the fuzzy membership between learning resources and knowledge points. Ferreira-Satler et al. [15] constructed a user model using fuzzy ontology and designed a learning resource recommendation method on the basis of ontology reasoning. This method neglected fuzzy problems in learning resource division. Tambe et al. [16] designed a recommendation system on the basis of fuzzy logic and ontology method. This system recommended resources to users using ontology reasoning and fuzzy logic in accordance with the inquiry words input by users. However, the system did not construct models for learning resources and learners. Sabitha et al. [17] clustered learning resources in accordance with learning styles through fuzzy c-mean clustering method and recommended learning resources on the basis of the learners' learning style. This method also neglected the fuzziness in categorizing learning resources and the knowledge level of learners. Although these studies had slightly discussed fuzzy problems during learning resource recommendation, the fuzziness in categorizing learning resources and the knowledge level of learners remained.

Based on the literature review, the existing learning resource recommendation methods hardly consider fuzziness in categorizing learning resources and knowledge level of learners. Therefore, the fuzzy problems in learning resource recommendation are not solved properly in existing associated studies. This situation further causes the poor accuracy of learning resource and learner models, thus influencing the final recommendation results. Thus, a learning resource recommendation method based on fuzzy logic (RRMF) is proposed. This method is used to perform a fuzzy division of learning resources and compute the degree of membership of learning resources to different knowledge points. This method also indicates that one learning resource belongs to different knowledge points at various degrees. In subsequent sections, the knowledge level model of learners is constructed through the fuzzy cognitive diagnosis method, which obtains the knowledge point mastery degrees of learners. On this basis, the learning resource recommendation model is established. Finally, the candidate learning resource scores of target learners are predicted on 
the basis of the knowledge level of learners, and those learning resources that satisfy the score range are recommended to learners.

The remainder of this study is organized as follows. Section 3 constructs the learning resource model using fuzzy set theory and constructs the learner model through the fuzzy cognitive diagnosis method. On this basis, the learning resource recommendation model is constructed. The steps and experimental design of the recommendation algorithm are introduced. Section 4 introduces the experimental results and analysis. Section 5 presents the conclusions drawn from this study.

\section{Methodology}

\subsection{Learning resource modeling}

This section mainly introduces the construction of the learning resource model using fuzzy set theory, which is conducive to the subsequent construction of the learning resource recommendation model. In practical applications, one resource frequently belongs to various types at different degrees. For example, a movie may be categorized as a comedy, romance, and adventure simultaneously. Similarly, a learning resource may belong to multiple types of knowledge points, and one problem may investigate multiple knowledge points. Thus, the fuzzy division of learning resources is accomplished in accordance with fuzzy set theory. One learning resource may belong to multiple types of knowledge points at different degrees.

Zadeh [18] proposed fuzzy set theory. This theory expresses the "mutual" relationship between an element and a set using a membership function, rather than the "alternative" relationship of the ordinary set. Furthermore, this theory is mainly used to depict fuzzy phenomena in the objective world. A relevant definition of the fuzzy set is introduced in the following text.

Definition 1: (Fuzzy set) The fuzzy set $\underset{\sim}{A}$ on the domain of discourse $X$ is defined as $\underset{\sim}{A}=\left\{\left(\mu_{A}(x), x\right) \mid x \in X\right\}$, where $\mu_{A}(x)$ is a determined real number in the continuous interval $[0,1]$ and is called the membership of element $x$ to $\underset{\sim}{A}$.

Given the item $I_{j}(j=1, \ldots, M)$ and the category set $X=\left\{x_{1}, x_{2}, \ldots, x_{L}\right\}, \mu_{x_{k}}\left(I_{j}\right)$ is used to express the correlation between the item $I_{j}$ and category $x_{k}$. Moreover, $\mu_{x_{k}}\left(I_{j}\right)$ can be understood as the degree of membership of the item $I_{j}$ to the category $x_{k}$. Therefore, the association between $I_{j}$ and $x_{k}$ can be expressed as a $2 \mathrm{D}$ vector, that is, $X_{j}=\left\{\left(x_{k}, \mu_{x_{k}}\left(I_{j}\right)\right), k=1, \ldots, L\right\}$.

In the process of learning resource recommendation, the learning resource corresponds to the item $I_{j}$, and the knowledge point associated with learning resources corresponds to the category $x_{k}$. Therefore, $\mu_{x_{k}}\left(I_{j}\right)$ can be used to express the degree of membership of resource $I_{j}$ to the knowledge point $x_{k}$. The correlation between learning resources and different knowledge points can be expressed using a 2D vector $\left(x_{k}, \mu_{x_{k}}\left(I_{j}\right)\right)$. Common membership functions mainly include triangle, trapezoid and Gaussian functions, and exponential-like function [19]. In this study, the exponential-like function is applied to calculate the membership function $\mu_{x_{k}}\left(I_{j}\right)$. The exponential-like function attenuates quickly and thus conforms to the correlation features between learning resources and knowledge points. Equation (1) is used as the calculation formula. $\mu_{x_{k}}\left(I_{j}\right)$ satisfies the condition that the degree of membership is positively related to the correlation degree between learning resources and knowledge points. If a learning resource is independent of a knowledge point, then the corresponding degree of membership is 0 .

$$
\mu_{x_{k}}\left(I_{j}\right)=\left\{\begin{array}{llc}
\left(\frac{1}{2}\right)^{\lambda \mid L_{j}\left(r_{k}-1\right)} & \text { if } & 1 \leq r_{k} \leq\left|L_{j}\right| \\
0 & \text { if } & r_{k}>\left|L_{j}\right|
\end{array}\right.
$$

where $N=\left|L_{j}\right|$ is the number of knowledge points correlated with the learning resource $I_{j}, r_{k}$ is the position of the knowledge point $x_{k}$ in all knowledge points related to the learning resource $I_{j}$ and is given by domain experts, and $\lambda$ is the adjustment parameter.

\subsection{Learner modeling}

The knowledge level of learners changes continuously with the progress in learning. Such a change is a progressive process, which is accompanied by certain fuzziness. Hence, the knowledge point mastery degree of learners cannot be specified, and the degree of mastery is fuzzy. The knowledge level model of learners is constructed using the fuzzy cognitive diagnosis model (FuzzyCDF) proposed by $\mathrm{Wu}$ et al. [20]. On the basis of this model, the knowledge point mastery degree of learners can be known, and the learners' scores in the learning resource or problem can be predicted.

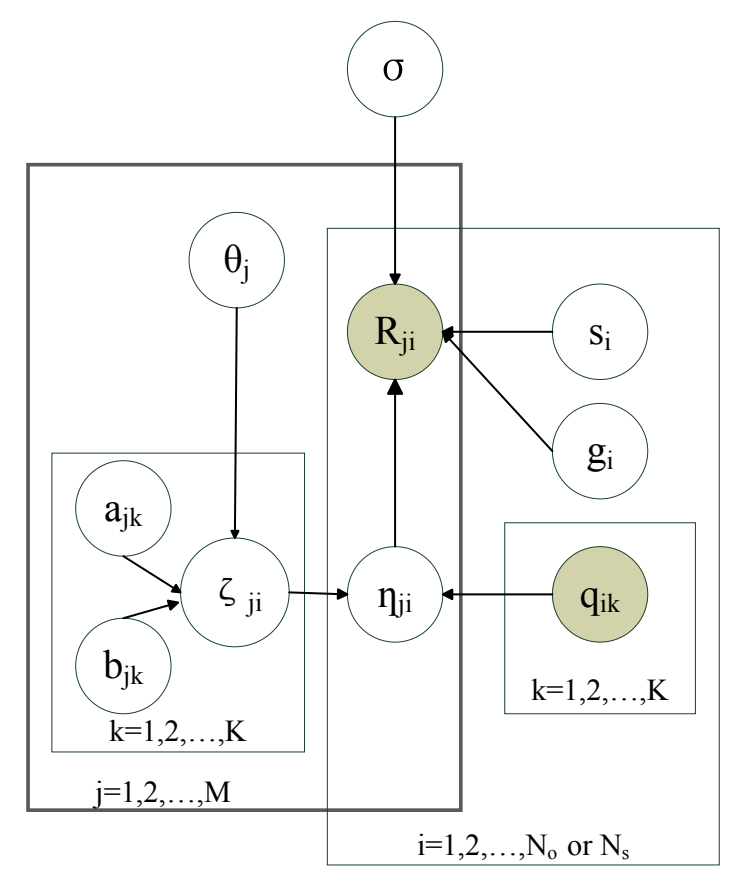

Fig. 1. Fuzzy Cognitive Diagnosis

FuzzyCDF overcomes the limitation of existing cognitive diagnosis models within objective problems, and 
FuzzyCDF can diagnose the performances (e.g., scores) of examinees in subjective and objective problems (Fig. 1). This model is a production process, which started with the latent traits of examinees. It determines the skill level of examinees initially and then calculates the knowledge mastery degree of examinees. Based on considerations of slip and guess factors, this model finally gains observable scores of examinees on different problems, where $\zeta_{j k}$ is the examinee with a latent trait of $\theta$. For the $k$ th skill (knowledge point) level (mastery degree), the logistic model can be expressed as follows:

$$
\zeta_{j k}=\mu_{k}(j)=\left(1+\exp \left(-1.7 a_{j k}\left(\theta_{j}-b_{j k}\right)\right)^{-1}\right.
$$

where $a_{j k}$ is the discrimination of problems, and $b_{j k}$ is the difficulty of problems. The performances of examinees can be calculated using Equation (3) or (4). The performances of examinees to subjective problems can be determined using Equation (3).

$$
P\left(R_{j i}=1 \mid \eta_{j i}, s_{i}, g_{i}\right)=\left(1-s_{i}\right) \eta_{j i}+g_{i}\left(1-\eta_{j i}\right)
$$

The performances of examinees to objective problems can be measured using Equation (4).

$$
\begin{aligned}
& P\left(R_{j i} \mid \eta_{j i}, s_{i}, g_{i}\right)= \\
& \quad \mathcal{N}\left(R_{j i} \mid\left(\left(1-s_{i}\right) \eta_{j i}+g_{i}\left(1-\eta_{j i}\right)\right), \sigma^{2}\right)
\end{aligned}
$$

where $R_{j i}$ is the score of examinee $j$ on the problem $i$, and $\eta_{j i}$ is the mastery degree of examinee $j$ on the problem $i$. If the knowledge points between each other on a problem are conjunctive, then the formula used is Equation (5). If the knowledge points between each other on a problem are compensatory, then the formula used is Equation (6). $s_{i}$ and $g_{i}$ denote the slip and guess factors of problem $i . \sigma^{2}$ is the variance of the normalized score of an examinee on a subjective problem.

$$
\begin{aligned}
& \eta_{j i}=\mu_{\cap I s k \leq K, q_{i k}=1} k(j) \\
& \eta_{j i}=\mu_{\mathrm{UIsk \leq K,q_{ik } = 1}} k(j)
\end{aligned}
$$

The parameters in the model were estimated using markov chain monte carlo algorithm. Detailed steps are introduced in reference [20].

The knowledge point mastery degree of learners can be gained using the FuzzyCDF. A learner can express a vector form related to a knowledge point. Moreover, the scores of learners on different problems, including scores on objective and subjective problems can be obtained.

\subsection{Similarity computation}

In the process of learning resource recommendation, one key problem is computing the similarity among learning resources and among learners. The learning resource is expressed in the vector form related to knowledge point, and thus the learner is expressed in knowledge point mastery degree, which can be expressed as a vector. Consequently, the similarities among different learning resources and among various learners were computed using cosine similarity calculation method based on the fuzzy set [19].

$$
\begin{aligned}
& \operatorname{sim}\left(I_{k}, I_{j}\right)= \\
& \frac{\sum_{i} \mu_{x_{i}}\left(I_{k}\right) \mu_{x_{i}}\left(I_{j}\right)}{\sqrt{\left(\left(\sum_{i}\left(\mu_{x_{i}}\left(I_{k}\right)\right)^{2}\right)\right)} \sqrt{\left(\left(\sum_{i}\left(\mu_{x_{i}}\left(I_{j}\right)\right)^{2}\right)\right)}} \\
& \operatorname{sim}\left(U_{k}, U_{j}\right)= \\
& \frac{\sum_{i} \mu_{x_{i}}\left(U_{k}\right) \mu_{x_{i}}\left(U_{j}\right)}{\sqrt{\left(\left(\sum_{i}\left(\mu_{x_{i}}\left(U_{k}\right)\right)^{2}\right)\right)} \sqrt{\left(\left(\sum_{i}\left(\mu_{x_{i}}\left(U_{j}\right)\right)^{2}\right)\right)}}
\end{aligned}
$$

Equation (7) is used to compute for the similarity among different learning resources. $\mu_{x_{i}}\left(I_{k}\right)$ is the membership degree of learning resource $I_{k}$ to the knowledge point $x_{i}$. Equation (8) is used to compute the similarity among different learners. $\mu_{x_{i}}\left(U_{k}\right)$ is the mastery degree of the learner $U_{k}$ to the knowledge point $x_{i}$.

\subsection{Learning resource recommendation model}

The learning resource recommendation model is constructed on the basis of the learning resource and the learner models. The overall framework of the constructed learning resource recommendation model is illustrated in Fig. 2. First, the fuzzy division of learning resources in accordance with a knowledge point is performed through the method introduced in Section 3.1. The membership degree of resources to different types of knowledge points can be gained. Second, the knowledge level model of learners is constructed through the fuzzy cognitive diagnosis method in Section 3.2 by determining the knowledge point master degree of learners. In addition, the neighbor users of the target learners are recognized using the formula for fuzzy similarity. Thus, the relevant knowledge point set that the target learner has to learn is obtained. Third, the knowledge points for recommendation are further screened on the basis of the knowledge level of the learner. Finally, the candidate learning resources, which are highly correlated with knowledge points for recommendation are identified, and the scores of the target learner on the candidate learning resources are predicted. The learning resources that satisfy the score range are recommended to the learner.

The proposed model can recommend complementing learning resources or new resources for subsequent learning in accordance with practical situations. Learners with relatively low knowledge level are generally offered complementing learning resources to compensate the learned but not mastered knowledge, whereas learners with a high knowledge level are generally recommended new learning resources to help progress their learning. These feats can be achieved by setting relevant parameters in the model. 


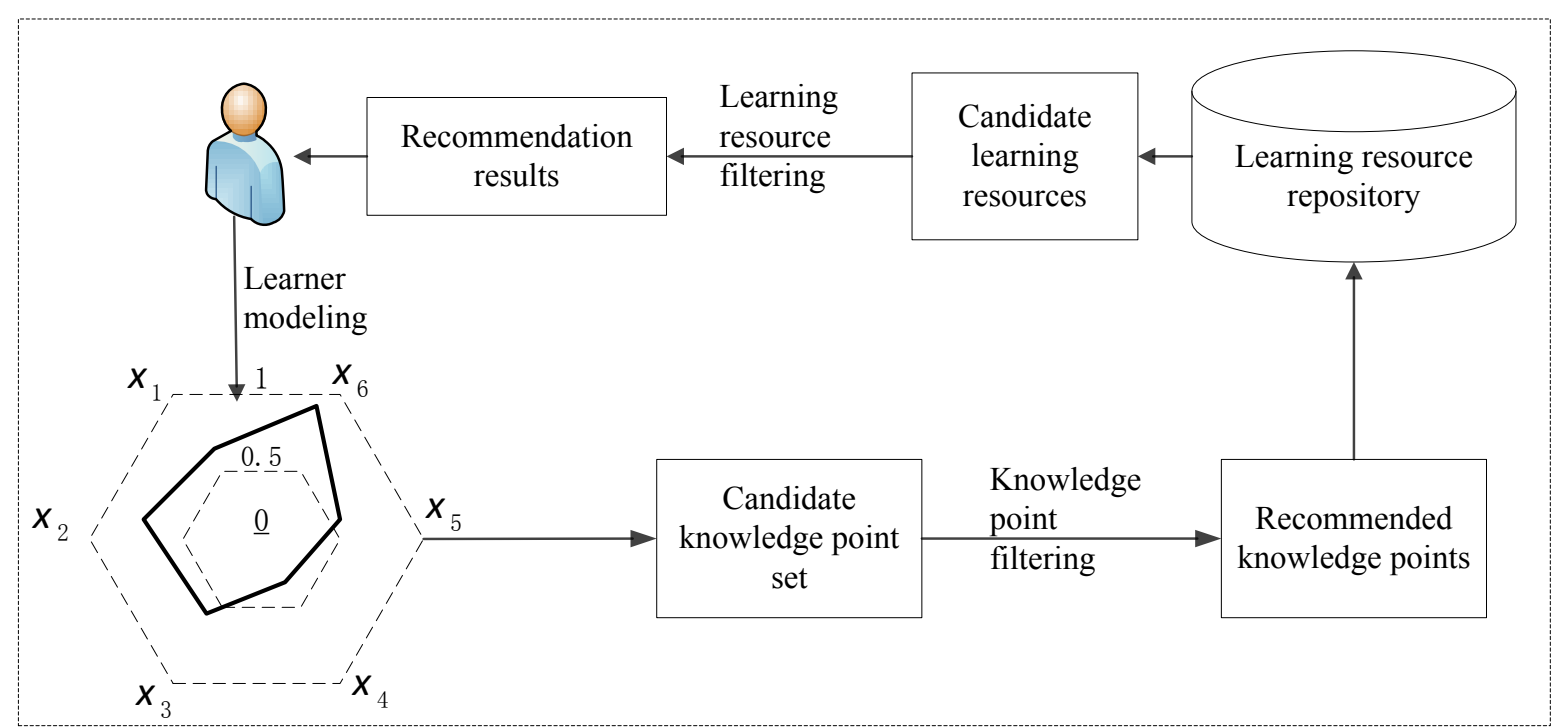

Fig. 2. Learning resource recommendation model

\subsection{Recommendation algorithm steps}

The specific algorithm steps are constructed on the basis of the learning resource recommendation model. In the learning resource recommendation, learning resources, especially problems, are mainly used to consolidate and increase the mastery degree of the learned knowledge points. Thus, these resources are generally those closely related to the knowledge points, which the target learner has not mastered well. In practical application, the priority is to determine the knowledge point set, which the target learner had not mastered well. The learning resource list, which highly correlates with the knowledge points in this set, is identified. Finally, the candidate learning resources with scores that satisfy the value range are recommended to learners. The recommendation algorithm steps are introduced as follows:

Input: correlation vector between learning resources and knowledge point, problems knowledge point correlation matrix, problem score, and the target learner.

Output: learning resource recommendation list.

(1) Based on the problem-knowledge point and the score matrixes, the mastery degrees of the target learner and other learners on different knowledge points can be computed using Equation (2).

(2) The neighbor users of the target learner are computed using Equation (8). On this basis, the knowledge point set that the target learner has to learn is further determined.

(3) Knowledge points that the target learner did not master well are screened from the knowledge point set, which is produced in Step (2) by setting a threshold.

(4) Candidate learning resources are matched with the knowledge points produced in Step (3). According to the setting of the threshold, learning resources, which are highly associated, are screened, thereby forming the candidate learning resource set.

(5) Scores of the learners on different candidate learning resources are further predicted using the cognitive diagnosis model FuzzyCDF.

(6) The top-N learning resources, which satisfy the score range, are recommended to the target learner, thus marking the end of the learning resource recommendation.

\subsection{Experiment design}

The proposed RRMF was compared with three methods on the basis of real datasets to verify the proposed RRMF effectively. The detailed contents are introduced as follows:

(1) Deterministic inputs, noisy, "and" gate (DINA) [21]. A cognitive diagnosis method models the examinees' skill proficiency and the slip and guess factors of problems with a Q-matrix. This method predicts the scores of learners on candidate resources and recommends those with high scores to the learner.

(2) Probabilistic matrix factorization (PMF) [22]. PMF decomposes a high-dimensional matrix into two lowdimensional matrixes from the perspective probability, thus predicting the performances of learners on problems and recommending relevant resources.

(3) Collaborative filtering (CF) [2]. CF is a resource recommendation method based on the preferences of neighbor users.

In this experiment, a real dataset introduced by $\mathrm{Wu}$ et al. [20] has been applied. The real dataset is composed of three parts, namely, FrcSub, Math1, and Math2. FrcSub is the problem dataset involving the addition and subtraction of fractions and is frequently used to verify the performance of the cognitive diagnosis model. FrcSub contains scores of learners on problems and knowledge point correlation matrix in problems. The former covers the score data of 536 learners on 20 objective problems, where 1 means right and 0 means wrong. The latter covers the relationships between 20 problems and 8 knowledge points, where 1 means related and 0 means unrelated. Math1 and Math2 are united mathematics examination datasets. These datasets are also composed of the score data of learners on problems and the problem-knowledge point correlation matrix. Specifically, Math1 covers scores of 4209 learners on 15 objective and 5 subjective problems. Scores of objective problems are expressed by 1 (correct) and 0 (wrong), whereas the scoring ratio of subjective problem ranges in the interval of $[0,1]$. The problem-knowledge point correlation matrix contains correlations between 20 problems and 11 knowledge points, where 1 means correlated and 0 means non-correlated. Math2 includes scores of 3911 learners on 16 objective and 4 subjective problems and correlations between 20 problems 
and 16 knowledge points. These datasets are summarized in Table 1.

Table. 1. Statistical information of the dataset

\begin{tabular}{lllll}
\hline Dataset & $\begin{array}{l}\text { Learne } \\
\text { r }\end{array}$ & $\begin{array}{l}\text { Objective } \\
\text { Problem }\end{array}$ & $\begin{array}{l}\text { Subjective } \\
\text { Problem }\end{array}$ & $\begin{array}{l}\text { Knowledge } \\
\text { Point }\end{array}$ \\
\hline Frcsub & 536 & 20 & 0 & 8 \\
\hline Math1 & 4209 & 15 & 5 & 11 \\
\hline Math2 & 3911 & 16 & 4 & 16 \\
\hline
\end{tabular}

The performances of the proposed RRMF on FrcSub, Math1, and Math2 are compared with those of the three other recommendation methods (DINA, PMF, and CF). First, each dataset is divided into the training and test sets randomly in accordance with different proportions. The proportions of a training set are fixed at $40 \%, 60 \%$, and $80 \%$, whereas the proportions of the corresponding test set are $60 \%, 40 \%$, and $20 \%$. Second, all recommendation methods are compared on the basis of the three proportions of datasets. Finally, the experimental results on the dataset whereby the training set accounted for $80 \%$ are used as the final experimental results.

In the experiment, the mean absolute error (MAE) is used as the performance evaluation metric of the algorithm. MAE is an evaluation metric, which is used frequently in the recommendation system. It tests the effect of the recommendation method by calculating the mean absolute difference between predicted scores and real value. A small MAE indicates an improved effect of the recommendation method. The formula for obtaining the MAE is expressed in Equation (9).

$M A E=\frac{\sum_{i=1}^{N}\left|p_{i}-q_{i}\right|}{N}$

where $N$ is the total number of scoring items of the learner, $p_{i}$ is the predicted score, and $q_{i}$ is the actual score.

\section{Result analysis and discussion}

The MAEs of different recommendation methods based on various datasets are listed in Table 2. For FrcSub, Math1, and Math2, the proposed RRMF is superior to others in terms of recommendation effect. Specifically, the MAEs are $7.82 \%, 13.08 \%$, and $17.82 \%$ lower in the RRMF for FrcSub than in the DINA, PMF, and CF, respectively. The MAEs are $10.34 \%, 15.18 \%$, and $16.29 \%$ lower in the RRMF for Math1 than in the DINA, PMF, and CF, correspondingly. The MAEs are $11.94 \%, 14.56 \%$, and $18.98 \%$ lower in the RRMF for Math2 than in the DINA, PMF, and CF, respectively. These findings indicate that the proposed RRMF is more effective than the DINA, PMF, and CF with respect to learning resource recommendations. Furthermore, DINA is quite unstable and show poor recommendation effect when the proportion of the training set is low. This phenomenon may be caused by the large diagnosis error of learners in terms of knowledge point mastery given the reduction of the training data. In comparison with the PMF, the advantage of the RRMF is unnoticeable when the test set proportion is low. However, the advantage of the RRMF becomes increasingly prominent with the increase in the training set proportion. In addition, the recommendation effect of the CF decreases dramatically with the reduction in the training set proportion. This observation is possibly due to the difficulty of using CF to recognize learners with the highest similarity through the conventional calculation method on a small dataset. The proposed RRMF adopts the fuzzy similarity calculation method to compute the similarity among learners and combines the knowledge level of the target learners. Therefore, the proposed RRMF achieves better recommendation effects than the other methods.

Table 2. Recommendation effects of the different methods

\begin{tabular}{|c|c|c|c|c|c|c|c|c|c|c|}
\hline \multirow{3}{*}{ Evaluation metric } & \multirow{3}{*}{ Methods } & \multicolumn{3}{|c|}{ FreSub } & \multicolumn{3}{|c|}{ Math1 } & \multicolumn{3}{|c|}{ Math2 } \\
\hline & & \multicolumn{3}{|c|}{ Training Ratio } & \multicolumn{3}{|c|}{ Training Ratio } & \multicolumn{3}{|c|}{ Training Ratio } \\
\hline & & $40 \%$ & $60 \%$ & $80 \%$ & $40 \%$ & $60 \%$ & $80 \%$ & $40 \%$ & $60 \%$ & $80 \%$ \\
\hline \multirow{4}{*}{ MAE } & DINA & 0.4903 & 0.3903 & 0.3827 & 0.5567 & 0.4567 & 0.3978 & 0.5791 & 0.4791 & 0.4011 \\
\hline & PMF & 0.4853 & 0.4853 & 0.4353 & 0.4562 & 0.4362 & 0.4462 & 0.4673 & 0.4273 & 0.4273 \\
\hline & $\mathrm{CF}$ & 0.5527 & 0.4927 & 0.4827 & 0.5673 & 0.4673 & 0.4573 & 0.5815 & 0.4815 & 0.4715 \\
\hline & RRMF & 0.3939 & 0.3739 & 0.3045 & 0.3844 & 0.3344 & 0.2944 & 0.3807 & 0.3417 & 0.2817 \\
\hline
\end{tabular}

A contrastive experiment of several methods for various datasets with different proportions of the training set is conducted to exhibit the advantages of the proposed RRMF intuitively. The results are depicted in Figs. 3-5. The experimental results when the proportions of the training set are $40 \%, 60 \%$, and $80 \%$ are demonstrated in Figs. 3, 4, and 5, correspondingly. The MAEs of all recommendation methods decrease with the increase in the proportion of the training set. This finding reflects that all recommendation methods are effective when the training set reaches a certain proportion. The MAEs of all the recommendation methods are not very small on the dataset wherein the proportion of the training set is $40 \%$ (Fig. 3). The model parameters in DINA, PMF, and RRMF must be deduced depending on the training set, and thus the small proportion of the training set may result in poor training accuracy of the model parameters and influence the final recommendation effect. However, the experimental results on the dataset under $60 \%$ of the training set are better than those under $40 \%$ (Fig. 4). Further analysis shows that the MAEs of all recommendation methods on the dataset under $80 \%$ of the training set are low. The RRMF possesses significant superiorities to all the other recommendation methods (Fig. 5) because the RRMF constructs the learner model through the fuzzy cognitive diagnosis method, which can accurately reflect the knowledge point mastery of learners. Moreover, it groups learning resources using fuzzy set theory, which describes the relationship between the learning resource and knowledge point accurately. Consequently, the RRMF assures the accuracy of the recommendation results. By contrast, the DINA only reflects whether learners master the knowledge points or not. The PMF and CF disregard the knowledge point mastery of learners, thereby resulting in the poorer performance of MAE than that of the RRMF. In particular, the RRMF is significantly advantageous on the large Math1 and Math2, thus further verifying the validity of the proposed RRMF. 


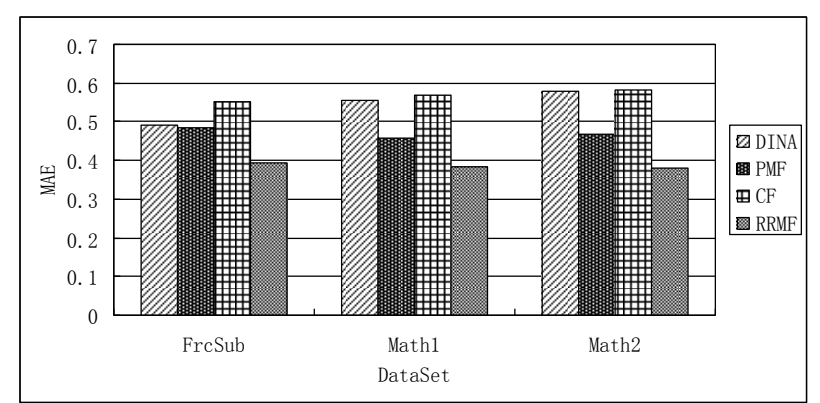

Fig.3. Experimental results of the training ratio of $40 \%$

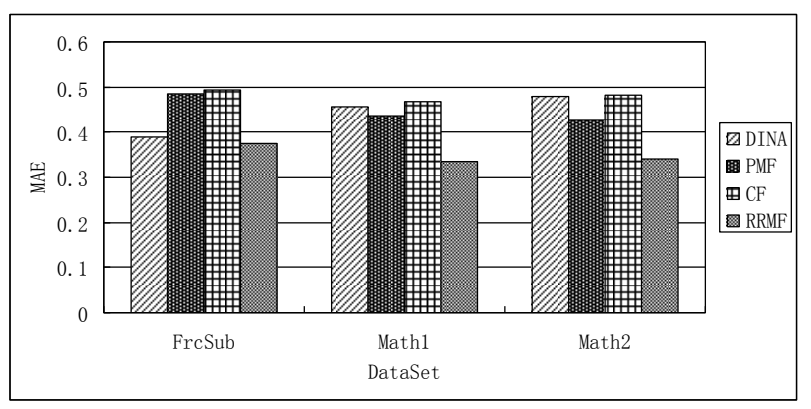

Fig.4. Experimental results of the training ratio of $60 \%$

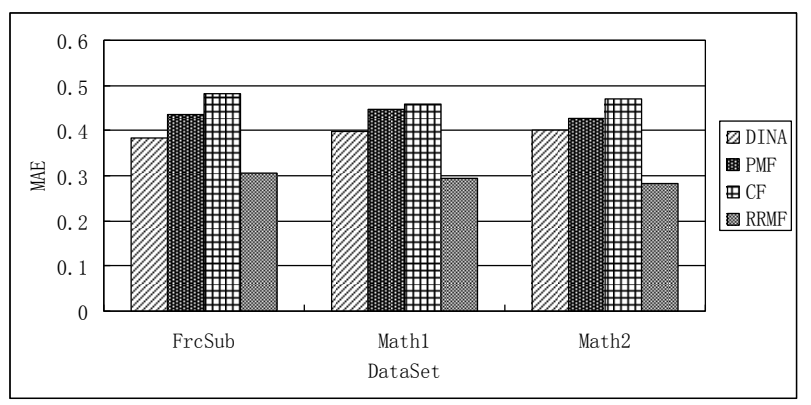

Fig. 5. Experimental results of the training ratio of $80 \%$

In addition, the real data of the actual examinations are applied as the dataset in this study. Each learner in the dataset has participated in all exams. Therefore, the sparsity of dataset is low. In actual applications, each learner can only browse and learn certain learning resources with limited efforts considering the high number of learning resources. Thus, the sparsity of data is typically high. Under this circumstance, conventional recommendation methods, such as CF, may offer poor recommendation results. Based on the experimental results in Table 2 and Figs. 3-5, the proposed RRMF is the optimum method because it simultaneously provides consideration to the similarity of learners (universality) and knowledge point mastery degree of learners (personality).

\section{Conclusions}

Fuzzy problems in categorizing learning resources and knowledge level of learners during learning resource recommendation might decrease the recommendation accuracy of online learning resources. A learning resource recommendation method was proposed in this study to address these fuzzy problems, overcome their influence on recommendation results, and increase the accuracy of recommendation. A contrastive experimental study of the proposed RRMF and other recommendation methods based on real datasets was conducted, thereby verifying the validity of the proposed RRMF. The following major conclusions were drawn:

(1) A learning resource model was accurately constructed using fuzzy set theory. This model can accurately describe the relationship between learning resources and the type of knowledge points, solve the fuzzy problem in categorizing learning resources, and assure the reliability of recommendation results.

(2) The knowledge level model of learners was constructed using fuzzy cognitive diagnosis. This method of diagnosis depicts the actual knowledge level of learners, assures the accuracy of recommendation results, and makes the recommendation results slightly explainable.

(3) The proposed RRMF can handle fuzzy problems effectively. This method is applicable to recommending learning resources because it can increase the accuracy of recommendation and improve performance recommendation.

The proposed RRMF can be used to recommend not only learning resources but also courses and learning paths in the field of education. However, this study has several limitations. The problem of same membership was not considered when one learning resource is associated with multiple knowledge points. This limitation will become a key research topic in the future.

\section{Acknowledgements}

The authors are grateful for the support provided by the Natural Science Foundation of China (Grant Nos: 71461013 and 61262033) and the Humanities and Social Science Research in Colleges and Universities of Jiangxi Province (Grant No: TQ1303).

This is an Open Access article distributed under the terms of the Creative Commons Attribution License

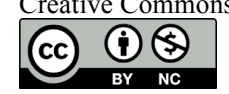

\section{References}

1. Manouselis, N., Drachsler, H., Vuorikari, R.,Hummel, H.,Koper, R., "Recommender systems in technology enhanced learning". In: Proceedings of the 16th ACM SIGKDD International Conference on Knowledge Discovery and Data Mining, Washington, DC, USA: ACM, 2011, pp.387-415.

2. Tan, H., Guo, J., Li, Y., "E-learning recommendation system". In: 2008 International Conference on Computer Science and Software Engineering, Wuhan, China: IEEE, 2008, pp.430-433.

3. Upendran, D., Chatterjee, S., Sindhumol, S., Bijlani, K., "Application of predictive analytics in intelligent course recommendation”. Procedia Computer Science, 93(1),2016,pp.917-923.
4. Chen, W., Niu, Z., Zhao, X., Li, Y., "A hybrid recommendation algorithm adapted in e-learning environments". World Wide Web, 17(2), 2014, pp.271-284.

5. Rodríguez, P. A., Ovalle, D. A., Duque, N. D., “A student-centered hybrid recommender system to provide relevant learning objects from repositories". In: Proceedings of the Second International Conference on Learning and Collaboration Technologies, Los Angeles, CA, USA: Springer, 2015, pp.291-300. 
6. HL dos Santos H. L., Cechinel, C., Araujo, R. M., Sicilia, M., "Clustering learning objects for improving their recommendation via collaborative filtering algorithms". In: Proceedings of the 9 th Research Conference on Metadata and Semantics Research, Manchester, UK: Springer, 2015, pp.183-194.

7. Klašnja-Milićević, A., Vesin, B., Ivanović, M., Budimac, Z., "ELearning personalization based on hybrid recommendation strategy and learning style identification". Computers \& Education, 56(3), 2011,pp.885-899.

8. Lu, J., “A personalized e-learning material recommender system". In: Proceedings of the 2nd International Conference on Information Technology for Application, Harbin, China: IEEE, 2004, pp.374-379.

9. Wan, S., Niu, Z., "A learner oriented learning recommendation approach based on mixed concept mapping and immune algorithm". Knowledge-Based Systems, 103(3), 2016, pp.28-40.

10. Chen, Y. H., Tseng, C. H., Huang, C. L., Deng, L.Y.,Lee, W. C., "Recommendation system based on rule-space model of two-phase blue-red tree and optimized learning path with multimedia learning and cognitive assessment evaluation". Multimedia Tools and Applications, 76(18), 2017, pp.18237-18264.

11. Liu, Z. B.,Song, W. A., Kong, X. Y.,Li, H. Y., "Research on Learner Modeling and Learning Resources Recommendation in Cloud Environment". e-EDUCATION RESEARCH, 38(07), 2017,pp.58-63.

12. Bernacki, J., "Recommending learning material in Intelligent Tutoring Systems ". Annales Universitatis Mariae Curie-Sklodowska. Sectio AI, Informatica, 16(1),2016,pp.1-11.

13. Salehi, M., Kamalabadi, I. N., "Hybrid recommendation approach for learning material based on sequential pattern of the accessed material and the learner's preference tree". Knowledge-Based Systems, 48(1),2013,pp.57-69.
14. Wu, D., Lu, J., Zhang, G., "A fuzzy tree matching-based personalized e-learning recommender system ". IEEE Transactions on Fuzzy Systems, 23(6),2015,pp.2412-2426.

15. Ferreira-Satler, M., Romero, F. P., Menendez-Dominguez, V. H., Zapata, A., Prieto, M. E., "Fuzzy ontologies-based user profiles applied to enhance e-learning activities". Soft Computing, 16(7), 2012, pp.11291141 .

16. Tambe, S. S., Kadam, G. V., "An Efficient framework for ELearning Recommendation system using fuzzy Logic and Ontology". International research journal of engineering and technology, 3(06),2016,pp.2062-2067.

17. Sabitha, A. S., Mehrotra, D., Bansal, A., "Delivery of learning knowledge objects using fuzzy clustering ". Education and information technologies, 21(5), 2016,pp.1329-1349.

18. Zadeh, L. A., "Fuzzy Sets". Information and Control, 8(3),1965,pp. $338-353$.

19. Zenebe, A., Norcio, A. F., "Representation, similarity measures and aggregation methods using fuzzy sets for content-based recommender systems". Fuzzy sets and systems, 160(1),2009,pp.76-94.

20. Wu, R., Liu, Q., Liu, Y., Chen, E., Su, Y., Chen, Z., Hu, G., "Cognitive Modelling for Predicting Examinee Performance". In: Proceedings of the 24th International Joint Conference on Artificial Intelligence, Buenos Aires, Argentina: AAAI Press, 2015, pp.10171024.

21. De La Torre, J., "The generalized DINA model framework". Psychometrika, 76(2),2011,pp.179-199.

22. Salakhutdinov, R., Mnih, A., "Probabilistic matrix factorization". In Proceedings of the 21st International Conference on Neural Information Processing Systems, Vancouver, B.C., Canada: ACM, 2008, pp.1257-1264. 